LAUR 99-5873

\title{
Testing of a Practical Low Pressure Permeator
}

\author{
David W. Howard \\ Westinghouse Savannah River Co. \\ R. Scott Willms and Stephen A. Birdsell \\ Los Alamos National Laboratory
}

\begin{abstract}
Palladium membrane permeators have long been used in tritium processing to separate gas mixtures into a pure-hydrogen permeate fraction, and a hydrogen-depleted retentate fraction. However, the retentate fraction has previously always required further purification (generally by oxidation followed by adsorption) to reach tritium concentrations low enough for environmental release. This report describes the development and testing of a practicalscale, low-pressure permeator to produce a hydrogen-depleted retentate fraction that can be stacked directly to the environment without further processing. This performance is attained by linking the permeator to a high vacuum system and by constructing the permeator with high vacuum conductance. The permeator incorporated three $1 / 8$ " $\mathrm{Pd} / \mathrm{Ag}$ tubes in a 6" shell. The pumping train consisted of turbomolecular pump, followed by a diaphragm pump. For this phase of testing only $\mathrm{H}_{2}$ performance was measured with tritium operation planned for later. This device has been tested with total feed flowrates up to 20 SLPM and various feed hydrogen concentrations. Permeate pressures were in the $10^{-5}$ to $10^{-2}$ torr range. Temperatures used were 350 to $450^{\circ} \mathrm{C}$. Presented are the design features of the permeator, and the results of testing this prototype device. Retentate hydrogen concentrations in the low ppm range were routinely achieved.
\end{abstract}

\section{INTRODUCTION}

Palladium (or Palladium/Silver alloy) permeators, have been employed in tritium processing operations for many years to remove hydrogen isotopes $\left(\mathrm{Q}_{2}\right)$, i.e., protium, deuterium, and tritium from other gases. Practically, $\mathrm{Pd} / \mathrm{Ag}$ is only permeable to $\mathrm{Q}_{2}$; a gas mixture is fed to one side of a $\mathrm{Pd} / \mathrm{Ag}$ membrane and a vacuum is applied to the other side of the membrane. The difference in hydrogen isotope partial pressure across the membrane is the driving force that generates a flow of Q2 from one side to the other. To date such devices have been used extensively to reduce the Q2 partial pressure in the retentate gas down to around 1 torr. This is insufficient for stacking the resulting gas if tritium was the isotope. However, previous work [1] suggested that a $\mathrm{Pd} / \mathrm{Ag}$ permeator could be used effectively at much lower partial pressures. The first direct test of this concept was with a single-tube permeator (10.9" long, $0.125^{\prime \prime}$ outer diameter, 0.003 " wall). This proof of principle experiment showed that the retentate and permeate hydrogen partial pressures could be brought to equilibrium at flowrates which were readily scalable to practical conditions [2]. Using these results as the basis, a practical-scale (higher feed flowrate, increased permeation rate, lower hydrogen concentration in the retentate, or some combination of these) low-pressure permeator was designed, constructed and tested. The results of testing this practical-scale permeator are presented here.

\section{EXPERIMENTAL APPARATUS}

Permeator: The permeator tested was manufactured by Resource Systems, Inc. of East Hanover, NJ, to patented design modifications of a commercial RSI-100 permeator. Figure 1 shows a diagram of the permeator.

The permeator consists of three helices of thin-wall palladium/silver tubing ( $1 / 8$ " OD x 0.003 " wall x 45 " length ea.), each of which is supplied from a common feed plenum by a long stainless-steel tube which runs down the length of the permeator axis before discharging into the palladium tubing. The helices in the low-pressure design are spaced on a $1 / 2$ inch pitch. This is intended to reduce the pressure of the permeated hydrogen in the exterior vicinity of each turn. The helices are supported on a perforated (63\% open area) cylinder, which permits the permeated hydrogen to flow radially inward (as well as outward) away from the permeator tubes. The axis of each helix is positioned on a radius within the permeator shell (chandelier-like). The helices are contained within a vacuum shell 6 " nominal OD x 20 " long. The permeate exhaust port consists of the entire 6 inch nominal diameter and is closely coupled to the inlet port of a turbomolecular pump. Heating is accomplished with 3 independent exterior clamp-on heaters around the shell instead of the conventional heater rods on the axis of each helix. All materials in contact with the gas are stainless steel, $\mathrm{Pd} / \mathrm{Ag}$, or the proprietary braze material joining the $\mathrm{Pd} / \mathrm{Ag}$ tubes to the stainless steel feed and retentate exhaust tubes.

The design features such as tube spacing and configuration, shell diameter, external heating, close-coupling with the turbomolecular pump and perforated helix support are all included to increase as much as reasonable the vacuum conductance from the $\mathrm{Pd} / \mathrm{Ag}$ tube surfaces to the pump inlet. This should produce the lowest reasonable pressure on the outside of the $\mathrm{Pd} / \mathrm{Ag}$ tubes and ultimately translate into low hydrogen partial pressures in the retentate. 


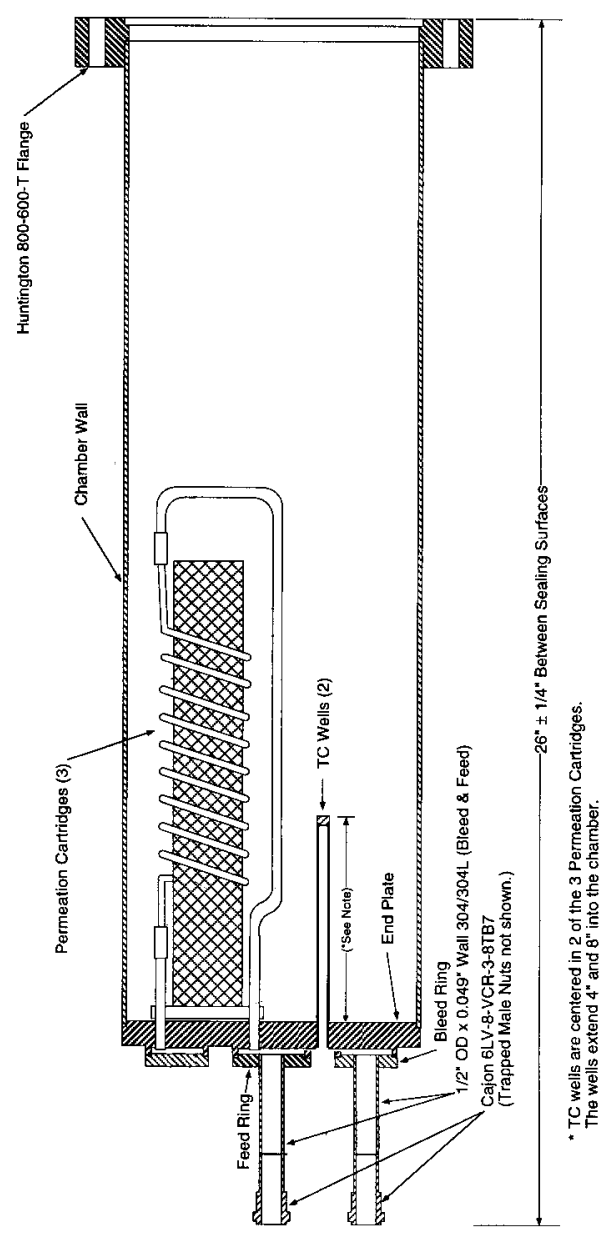

Figure 1 Low Pressure Permeator resulting in a fixed permeate pressure, easing the data interpretation. For each flowrate combination the pressures, temperature, flowrates and gas composition were recorded when conditions reached steady state (generally about 2 hours after changing input conditions). GC measurements were repeated a minimum of 5 measurements for each run.

Using the retentate gas composition and total pressure, the retentate $\mathrm{H}_{2}$ partial pressure could be calculated. Since the permeate was only $\mathrm{H}_{2}$, the shell-side $\mathrm{H}_{2}$ partial pressure was the same as the total vacuum pressure. The vacuum pressure was measured after a right-angle elbow connected to the permeator shell; the temperature at that point was measured to be approximately $34^{\circ} \mathrm{C}$. Also this cold cathode gauge was calibrated for $\mathrm{N}_{2}$ rather than the $\mathrm{H}_{2}$ gas of the permeate. Thus, the following correction was applied to determine the permeate pressure at the membrane:

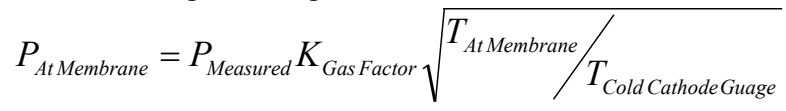

The cold cathode gauge manufacturer listed the gas factor as 0.43 . No attempt was made to correct for conductance pressure losses, but order-of-magnitude calculations have indicated this to be negligible.

\section{RESULTS}

Figure 3 shows a set of typical results obtained by feeding a constant flow of about $10 \mathrm{sccm}$ of hydrogen to the permeator mixed with nitrogen of feed rates varying from 25 sccm to $19,000 \mathrm{sccm}$ (hydrogen feed concentrations from $30 \%$ to $500 \mathrm{ppm}$ ) at $360^{\circ} \mathrm{C}$. The permeate pressure (permeator shell-side pressure) remained essentially constant at about $6 \times 10^{-4}$ torr, while the retentate partial pressure averaged about $2 \times 10^{-3}$ torr at the lower flow rates. However, at feed rates exceeding $2000 \mathrm{sccm}$ the retentate partial pressure increased dramatically above the permeate pressure. This point, at which the retentate and permeate partial pressures begin to differ, is referred to as "breakthrough". At higher flowrates, ever-increasing amounts of hydrogen remain in the retentate. It is clear that significant non-equilibrium exists between the retentate and permeate partial pressures at flow rates exceeding $2000 \mathrm{sccm}$, but the state of equilibrium below breakthrough is unclear.

Test Manifold: Feed gases (hydrogen and nitrogen) were injected using Brooks 5850EM mass flow controllers supplied from gas cylinders. Outlet retentate flow was measured with a Brooks 5850EM mass flow meter. The pressure of the feed and retentate were measured with Baratron transducers. The permeate vacuum pressure was measured with a cold cathode gauge. Feed and retentate compositions were measured by an MTI model M200 gas chromatograph using a $10 \mathrm{~m}$ molecular sieve column and a thermal conductivity detector (TCD). Calibration showed that the TCD could detect down to $0.3 \mathrm{ppm} \mathrm{H}_{2}$. The discharge pressure of the retentate was controlled using a backpressure regulator. A Varian Turbo-V250 turbomolecular pump $\left(250 \mathrm{l} / \mathrm{sec} \mathrm{N}_{2}, 200 \mathrm{l} / \mathrm{sec} \mathrm{H}_{2}\right)$ backed by a diaphragm pump was used to maintain the permeator shellside vacuum. The overall experimental setup is shown on Figure 2.

Procedure: The shell of the permeator was pumped down and the permeator heated to and maintained at a fixed operating temperature between 350 and $400^{\circ} \mathrm{C}$. Two types of test were performed: 1) a fixed flowrate of $\mathrm{H}_{2}(0.4-135$ sccm) was maintained during an experimental run, while the flowrate of the nitrogen was varied (30 - 20,000 sccm); or 2) the feed $\mathrm{H}_{2}$ composition was maintained at a fixed value (25 ppm - 30\%) during the experimental run, while the total feed flowrate was varied $(30-20,000 \mathrm{sccm})$. The first procedure using a fixed $\mathrm{H}_{2}$ flowrate was more convenient, as a fixed load of permeate was sent to the turbomolecular pump,

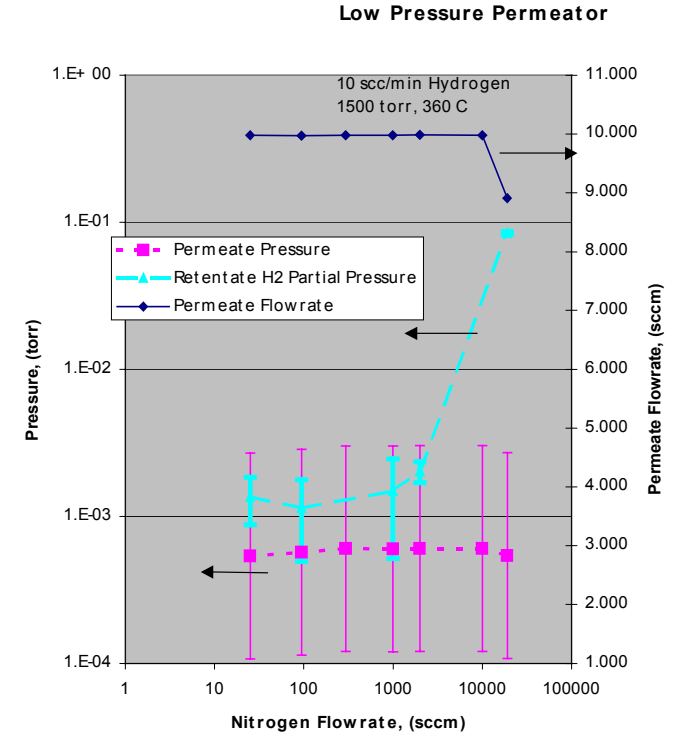

Figure 3 Typical Results from Low Pressure Permeator 
Figure 4 shows a set of typical results where the conclusions are more clear-cut. These measurements fed a constant flow of about $1.5 \mathrm{sccm}$ of hydrogen to the permeator mixed with nitrogen of feed rates varying from $25 \mathrm{sccm}$ to about $7000 \mathrm{sccm}$ (hydrogen feed concentrations from about $6 \%$ to $225 \mathrm{ppm}$ ) at a temperature of $400^{\circ} \mathrm{C}$. The permeate pressure (permeator shell-side pressure) remained essentially constant at about $1 \times 10^{-4}$ torr, but the retentate partial pressure was more than an order of magnitude higher, averaging about $5 \times 10^{-3}$ torr, until feed rates exceeded 2000 $\mathrm{sccm}$ at which point the retentate hydrogen partial pressure increased even further. The hydrogen partial pressure in the retentate corresponds to a hydrogen concentration of about $1.5 \mathrm{ppm}$, below $2000 \mathrm{sccm}$ feed flowrate.

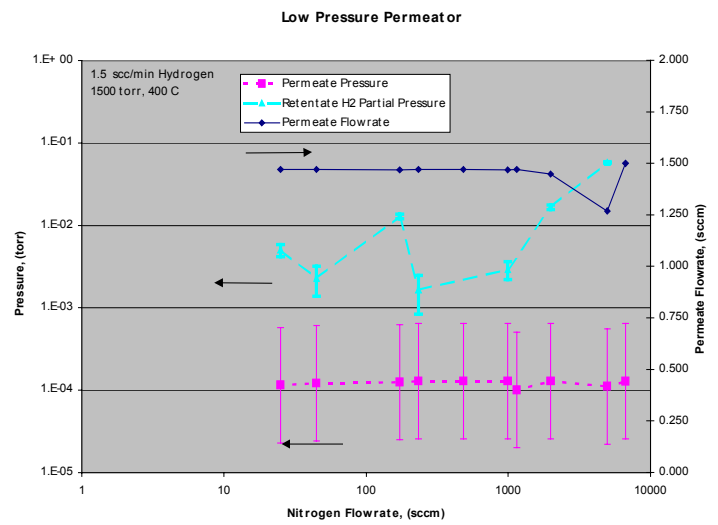

Figure 4 Permeator Performance at $400^{\circ} \mathrm{C}, 1500$ torr, and $1.5 \mathrm{sccm}$ Hydrogen Feed

For this experimental run it is clear that the retentate partial pressure of hydrogen is NOT in equilibrium with the permeate pressure; there is a significant difference in partial pressures between the retentate and the permeate (shell-side). The error bars on the data points show the estimated experimental uncertainty in the measurements. The difference in the partial pressures exceeds the uncertainty. The behavior of the differential between the retentate hydrogen partial pressure and the permeate pressure shown here is typical of all conditions tested.

\section{DISCUSSION}

Prior to these tests, there was concern that a permeator would be unable to remove hydrogen isotopes from other gases to the extent that would allow direct venting of the retentate gas when operating at a "practical" flowrate. A criteria for direct venting is often set at less than $5 \mathrm{ppm}$ of tritium, and "practical" flowrates of 1 - 10 liters per minute are often required. These tests have conclusively demonstrated that very low hydrogen concentrations $(1-5$ $\mathrm{ppm}$ ) can be reached at flowrates up to several 10's of liters per minute in a small permeator. This work demonstrates the practicality of a permeator system for such applications, and provides the basis for designing such permeators. Such a system would probably employ 1 or more "conventional" high-pressure permeators using higher-capacity, higherpressure vacuum pumps upstream of this low-pressure permeator. Such a system would remove the bulk of the hydrogen in the first stages, and use the low-pressure permeator to "clean-up" the retentate to levels suitable for venting. Such multi-stage permeator systems for several applications are currently in early stages of design.

\section{CONCLUSION}

These experiments demonstrate the practicality of this permeator as part of system for applications to produce a very high purity retentate which can be directly vented, or to remove large quantities of tritium from very high flow rate feeds. It was found that this type of permeator could produce a retentate containing less than $5 \mathrm{ppm}$ of hydrogen at flowrates up to $20,000 \mathrm{sccm}$. Similar results were obtained at temperatures from $360-440{ }^{\circ} \mathrm{C}$, and permeate pressures from $10^{-6}$ to $10^{-2}$ torr.

\section{REFERENCES}

[1] S. A. Birdsell, R. S. Willms and R. C. Wilhelm; "UltraHigh Tritium Decontamination Of Simulated Fusion Fuel Exhaust Using A 2-Stage Palladium Membrane Reactor", Proceedings of the ANS 12th Topical Meeting on Technology of Fusion Energy, Reno, June 16-20, 1996

[2] Willms, R. Scott, Pamela R. Arzu, Kevin G. Honnell and Stephen A. Birdsell; "Initial Testing Of A Low Pressure Permeator For Tritium Processing"; Proceedings of the Fifth International Symposium on Fusion Nuclear Technology, Rome, September 19-24, 1999. 
LAUR 99-5873

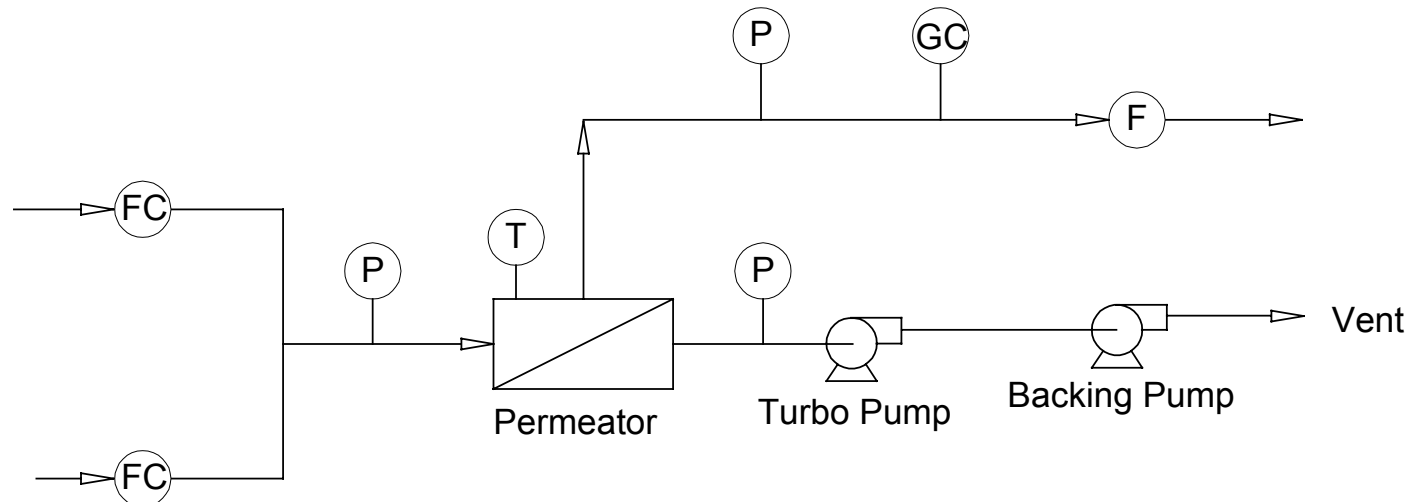

Figure 2 Experimental Setup 